\title{
Pre-hospital management and risk factors in children with acute diarrhoea admitted to a short-stay ward in an urban South African hospital with a high HIV burden
}

\author{
M L Cooke, MB ChB, FCPaed; E D Nel, MB ChB, MMed, FCP, BSc (Hons); \\ M F Cotton, MB ChB, FCPaed, MMed (Paed), PhD, DCH, DTM\&H
}

Department of Paediatrics and Child Health, Faculty of Medicine and Health Sciences, Stellenbosch University, Parow, Cape Town, South Africa

Corresponding author: M L Cooke (louise.cooke@uct.ac.za)

\begin{abstract}
Background. Diarrhoea remains a major cause of childhood morbidity and mortality in the developing world. Implementation of World Health Organization Integrated Management of Childhood Illness (IMCI) guidelines and pre-hospital use of oral rehydration therapy (ORT) in the Western Cape Province of South Africa are not well described.

Objectives. To document pre-hospital home and primary care management of diarrhoea, and certain risk factors and complications of diarrhoea.

Methods. We used a prospective descriptive convenience sample of children admitted to the short-stay ward at Tygerberg Hospital, Parow, Cape Town, between 1 February 2007 and 31 May 2008. Caregivers were interviewed, and demographic, clinical and laboratory variables were collected.

Results. We recruited 142 children, median age 8.9 months. A third had moderate malnutrition. Twenty-four (16.9\%) were HIV-exposed, with $9(6.3 \%) \mathrm{HIV}$-infected. HIV-exposed children were significantly younger than unexposed children $(p=0.03)$. Weight-for-age $Z$-scores (WAZ) were significantly lower in HIV-infected than in HIV-exposed, uninfected children $(p=0.02)$. Eighty per cent of caregivers gave ORT and $35.2 \%$ stopped feeds. Only 1 of 43 children aged under 6 months was exclusively breastfed. Advice at primary care level rarely complied with IMCI guidelines.

Conclusions. Most caregivers do give ORT, but advice given at primary care level is often suboptimal. Many hospitalised children with diarrhoea are malnourished. Children with HIV infection are at increased risk of diarrhoeal disease and malnutrition, and HIV exposure appears to increase the risk of early presentation with diarrhoea. Ongoing strategies are needed to ensure optimal prevention policies, prehospital management and nutritional rehabilitation.
\end{abstract}

S Afr J CH 2013;7(3):84-87. DOI:10.7196/SAJCH.472

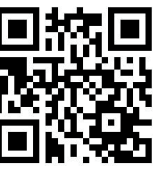

Diarrhoea remains a major cause of morbidity and mortality in children, especially in developing countries. It is the second most common cause of death in children under 5 years of age and is responsible for 1.4 million deaths each year. ${ }^{[1,2]}$ The Medical Research Council Burden of Disease Report indicates that diarrhoeal disease is responsible for over 10000 deaths annually in South Africa ${ }^{[3]}$ South Africa is one of the countries where the infant mortality rate has increased between 1990 and 2008, with no progress towards achieving Millenium Development Goal 4 by 2015. ${ }^{[4]}$

In 1980 the World Health Organization (WHO) initiated its programme for control of diarrhoeal disease (CDD), aiming to reduce morbidity and mortality, with the primary intervention being promotion of oral rehydration therapy (ORT) ${ }^{[5]}$ Despite initial success and reduction in mortality rates, there has been stagnation in progress rowards optimal management of diarrhoeal disease in low- and middle-income countries, with many children not receiving ORT, increased fluids or ongoing nutrition during episodes of acute diarrhoea ${ }^{[2,5,6]}$ Research on the diseases responsible for the highest number of deaths in children has not been sufficiently prioritised..$^{[2,7]}$

WHO Integrated Management of Childhood Illness (IMCI) guidelines on the management of diarrhoea stipulate that advice on correct feeding and fluid replacement and on danger signs must be provided by healthcare workers at each consultation. ${ }^{[8]}$ Primary healthcare provided at public clinics in the Cape Town Metropole has been based on IMCI principles since 2000. Home-made sugar-salt solution (SSS) composed of 1 litre of clean water with half a teaspoon of salt and 8 teaspoons of sugar as ORT is promoted on the Roadto-Health document and in dedicated ORT areas in clinics. There is, however, a paucity of data regarding the success of both IMCI and use of ORT in the Western Cape Province of South Africa.

Risk factors for diarrhoeal disease in our community are also poorly defined. Early studies have consistently shown an increase in length of hospitalisation and mortality in HIV-infected children in other parts of South Africa. ${ }^{[9,10]}$ Studies now indicate that HIVexposed uninfected infants also have increased infectious morbidity and mortality. ${ }^{[1-14]}$ Diarrhoea has been recognised as a nutritional disease for more than 2 decades, and malnutrition contributes to diarrhoeal disease morbidity and mortality. ${ }^{[15]}$

In the Western Cape there is an established prevention of motherto-child transmission (PMTCT) programme for HIV infection. Mothers are offered voluntary screening for HIV during pregnancy, antiretrovirals are given to mother and child if the mother is HIV-infected, and the mother is counselled in the choice between exclusive breastfeeding or replacement formula feeds. 


\section{Objectives}

To document pre-hospital home and primary care management of diarrhoeal disease, including use and mixing of ORT, fluid intake at home, and advice from healthcare providers in the context of the IMCI. We also aimed to identify potential risk factors and complications of diarrhoeal disease related to HIV status, nutritional status, feeding choice, sanitation and electrolyte disturbances.

\section{Methods}

This was a prospective descriptive study of children with diarrhoea admitted to the short-stay ward at Tygerberg Children's Hospital, Parow, Cape Town, between 1 February 2007 and 31 May 2008. The ward accommodates new referrals from general practitioners (GPs) and public clinics, and the hospital serves a population of low socioeconomic status. Children are admitted if dehydrated and if they failed a trial of ORT. Verbal consent was obtained by explaining that the information was anonymous and had no impact on the child's management, and consent was documented in the patient notes. The parent or caregiver was interviewed by a final-year medical student research assistant, under supervision of the principal investigator, using a structured questionnaire. This was a convenience sample depending on the availability of the research assistant. On the day of availability, all children in the ward with diarrhoea were included if consent was given. Responses were recorded on the questionnaire, then entered on a Microsoft Access database.

Data collected included demographic information; admission weight; home management of diarrhoea, including if ORT was given, whether it was in sachet form or SSS, and how it was mixed; whether increased fluids were given; whether normal feeds were continued; how long it took to seek medical assistance; what advice was given by the primary healthcare provider; and the number of times help was sought before hospital admission. We also documented feeding choice in children younger than 6 months over the preceding month; domestic water source; previous admissions for gastroenteritis; underlying HIV disease; and recorded electrolyte results in those who had blood tests. Electrolytes were measured at the National Health Laboratory Services according to standard laboratory procedure. HIV infection was confirmed by a positive enzymelinked immunosorbent assay (ELISA) together with a positive HIV polymerase chain reaction (PCR) test in children under the age of 18 months, or a second ELISA in older children. A child was classified as HIV-exposed if the mother was HIV-positive during the pregnancy, and as HIV-unexposed if the mother had documented negative status during the pregnancy. The HIV-exposed child was further classified as HIV-infected if PCR-positive; HIV-exposed, uninfected if the PCR test was negative; and HIV-exposed, unknown if no PCR had been done.

Descriptive statistics were used to describe the study population. Weight-for-age $Z$-scores (WAZ) were calculated using EpiInfo (Centers for Disease Control and Prevention, Atlanta, GA). As only admission weights were recorded, and weights after rehydration were not available, corrected (true) weight was estimated using the degree of dehydration as noted by the admitting clinician, with $5 \%$ or $10 \%$ being added to the admission weight depending on degree of dehydration.

\section{Statistical methods}

Statistica version 10 (Statsoft) was used to analyse the data. Continuous data were described as medians with upper and lower quartiles. Medians were compared using the Kruskal-Wallis ANOVA test. Categorical variables were compared using the Pearson chi-square and ML (maximum likelihood) chi-square tests in a contingency table.
Permission to conduct database-related research was obtained from the Human Research Ethics Committee of Stellenbosch University.

\section{Results}

We recruited 142 children between 1 February 2007 and 31 May 2008, representing $10.3 \%$ of the 1382 children admitted with diarrhoea during the 16-month period. One parent who was approached did not give consent. The demographic profile, HIV status and potential risk factors are set out in Table 1 . One-third (48/142) were moderately malnourished (WAZ $<-2$ standard deviations). The $24 \mathrm{HIV}$-exposed and HIV-infected children were significantly younger than the HIVunexposed children, with a median age of 6.1 months as opposed to 8.9 months $(p=0.03)$. The WAZ of the 9 children in the HIV-infected group (median -1.9) was significantly lower than that of the other 15 in the HIV-exposed group.(median 0.8) $(p=0.02)$

Most caregivers (112; 78.9\%) gave ORT at home before seeking help from a healthcare practitioner. Of these, 76 (67.9\%) gave homemade SSS, and 14 (12.5\%) mixed this incorrectly. Twelve used too much salt (range 1 - 2 teaspoons per litre of water), 2 used too much sugar, and in 4 cases all 3 components were incorrect. Fifty-two (36.6\% of the total) gave ORT and/or milk but no additional volume of daily fluid intake, and 50 (35.2\%) stopped their usual milk feeds and only gave other fluids, including ORT.

Caregivers visited a healthcare provider a mean of 2.5 days (range 1 - 14 days) after the onset of symptoms. Before admission to the hospital, 6 did not seek medical advice at primary level, 58 had 1 primary care visit, and 50 were referred after 2 visits to a healthcare

\section{Table 1. Profile of children admitted with diarrhoea $(N=142)$}

Age
Median

Range

Gender, $n(\%)$

Male

WAZ (corrected for dehydration) $(N=138)$

Median (range)

$\mathrm{WAZ}<-2, n(\%)$

$\mathrm{WAZ}>+2, n(\%)$

HIV status, $n(\%)$

HIV-unexposed

HIV-exposed

HIV-infected

HIV-exposed, uninfected

HIV-exposed, unknown

20 days - 64 months

$79(55.6)$

eeding choice $<6$ months $(N=43), n(\%)$

Exclusively breastfed

Mixed feeding

Formula only

Sanitation, $n(\%)$

Municipal water inside

89 (62.7)

Own outside tap

Shared outside tap

$-1.07(-1.94--0.05)$

32 (32.1)

$118(83.1)$

$9(6.3)$

$11(7.7)$
8.9 months

$\mathrm{WAZ}$ = weight-for-age $Z$-score. 
provider, 18 after 3 visits and 7 after 4 visits ( 3 unknown). Of the caregivers, $78.8 \%$ attended a free local clinic (for the first visit), while $12.7 \%$ went to GPs (fee for service) and $1.4 \%$ sought advice at a pharmacy (10 unknown). Table 2 shows the advice given by each group of health providers. There is an impression that the clinics gave appropriate advice regarding fluid intake, feeding, SSS recipe and danger signs more often than GPs, but none of the differences in advice given were statistically significant (Pearson and ML chi-square tests).

Twenty-one children (14.8\%) had had 1 previous admission for diarrhoea, 2 (1.4\%) 2 previous admissions, and 7 (4.9\%) more than 2 admissions.

Blood electrolytes were measured in $125 / 142$ children. Twenty (16.0\%) had hypernatraemia $\left(\mathrm{Na}^{+}>145 \mathrm{mmol} / \mathrm{l}\right)$, with a range of $146-169 \mathrm{mmol} / \mathrm{l}$. There was no significant increase in hypernatraemia in cases where SSS had been incorrectly mixed ( $p=0.6)$. Sixty-seven $(53.6 \%)$ of the tested children had hypokalaemia $\left(\mathrm{K}^{+}<3.5\right.$ $\mathrm{mmol} / \mathrm{l})$. There was no significant difference in potassium levels between those who used pre-packaged ORT sachets and homemade SSS $(p=0.45)$.

\section{Discussion}

This large prospective sample reflects the profile of the usual patient group admitted to the short-stay ward at our public hospital. The nutritional status of these children, with one-third moderately malnourished, was worse than the population average described in the National Food Consumption survey of 2005, which showed that $9 \%$ of South African children have WAZ $<-2 .{ }^{[16]}$ HIVinfected children are most underweight. These findings either demonstrate the negative impact of diarrhoea on nutrition or that nutritionally compromised children are vulnerable to diarrhoea, ${ }^{[15]}$ and support studies showing that HIV-infected children are nutritionally compromised. ${ }^{[9,11]}$

HIV-exposed children in our cohort presented with diarrhoea at a younger age than other children. Possible explanations include the lack of protective effect of breastfeeding and exposure to formula replacement feeds, deficient transplacental transfer of protective maternal antibodies, and increased exposure to pathogens from immunodeficient individuals in the household, exacerbated by crowding and compromised socioeconomic conditions associated with HIV infection. ${ }^{[12]}$ They represent a high-risk group.

The HIV exposure rate in our group is similar to that in the Western Cape seroprevalence survey $(15 \%) ;^{[17]}$ however, with a transmission rate of $5 \%$ quoted for the

Table 2. Advice given by healthcare provider at first visit

\begin{tabular}{llll}
\hline Advice given & $\begin{array}{l}\text { Primary clinic } \\
(\mathbf{N = 1 1 2})\end{array}$ & $\begin{array}{l}\text { GP } \\
(\boldsymbol{N}=18)\end{array}$ & $\begin{array}{l}\text { Pharmacist } \\
(\mathbf{N = 2})\end{array}$ \\
\hline Give extra fluid, $n$ (\%) & $57(50.8)$ & $8(44.4)$ & $1(50)$ \\
$\quad$ CI & $0.416-0.6015$ & $0.2149-0.6740$ & \\
Recipe for SSS, $n(\%)$ & $50(44.6)$ & $4(22.2)$ & $1(50)$ \\
$\quad$ CI & $0.3544-0.5385$ & $0.0302-0.4143$ & \\
Stop milk, $n(\%)$ & $20(17.9)$ & $3(16.7)$ & 0 \\
$\quad$ CI & $0.1076-0.2495$ & $0-0.3388$ & \\
Continue feeds including milk, $n(\%)$ & $19(17)$ & $4(22.2)$ & 0 \\
$\quad$ CI & $0.1001-0.2392$ & $0.0302-0.4143$ & \\
Danger signs of dehydration, $n(\%)$ & $4(3.6)$ & $1(5.6)$ & $1(50)$ \\
$\quad$ CI & $0.0013-0.0701$ & $0-0.1614$ & \\
& & & \\
GP = general practitioner; CI = confidence interval; SSS = sugar-salt solution. &
\end{tabular}

PMTCT programme in the Western Cape, we would only expect 1 - 2 HIV-infected children in a cohort of 142 , as opposed to 9. This finding supports findings of others that HIV-infected children are particularly vulnerable to diarrhoeal disease. ${ }^{[9-11]}$

The 2003 South Africa Demographic and Health Survey (SADHS) on 1859 mothers countrywide reported that 159 children had diarrhoea in the preceding 2 weeks. The survey showed that $60 \%$ of their caregivers gave ORT at home, $31 \%$ gave no ORT and only $24 \%$ increased fluids, while $40 \%$ reduced or stopped normal food intake. ${ }^{[18}$ The $2009 \mathrm{WHO} / \mathrm{UNICEF}$ report indicated that worldwide $42 \%$ of children received ORT, but only $22 \%$ received increased fluids and $30 \%$ were offered reduced amounts of food. ${ }^{[6]}$ Our finding that $80 \%$ of caregivers gave ORT is therefore very encouraging. A study at Red Cross War Memorial Children's Hospital, also in Cape Town, more than 10 years ago showed that despite $70 \%$ of caregivers having knowledge of ORT, only $9 \%$ gave it. ${ }^{[19]}$ The improvement probably reflects the success of the IMCI programme and SSS promotion implemented after that study. It is concerning that $18 \%$ of ORT solutions were incorrectly mixed. A study in the rural Western Cape also showed that although up to $90 \%$ of caregivers had knowledge of SSS, only $50 \%$ used it, and less than half mixed the solution correctly. ${ }^{[20]}$ There is risk associated with incorrect mixing, including hypernatraemia and salt poisoning, although this was not demonstrated as significant in our study. As in the SADHS, a third of our caregivers did not give more fluid volume than usual, indicating poor understanding of fluid losses in diarrhoea, and $35 \%$ of children had their normal feeds discontinued (replaced by ORT). This places children at risk of entering the vicious cycle of diarrhoea followed by malnutrition

Public health promotional material suggests that caregivers attend a primary care facility within a day of a child developing diarrhoea. We demonstrated a delay in seeking healthcare. Advice regarding use of ORT, feeding and danger signs was poor at both public clinics and GP rooms, putting children at potential risk.

Conclusions about bottle feeding as a risk factor or a protective effect from breastfeeding cannot be made from our cohort, as there was no control group of children without diarrhoea with similar feeding methods. However, the beneficial effect of breastfeeding has been demonstrated convincingly and repeatedly in the past, ${ }^{[2,22]}$ so it is important to note that of the children under 6 months of age, only 1 was exclusively breastfed. This is lower than the exclusive breastfeeding rate in South Africa of $8.3 \%{ }^{[18]}$ Breastfeeding is one of the components of the CDD prevention programme, and must be promoted locally.

Water, sanitation and hygiene are key elements in the prevention of diarrhoeal disease. ${ }^{[23]}$ Almost $40 \%$ of households in our study had to transport and store water from outside taps, raising concerns about hygiene and contamination of water, especially that used for formula preparation. Zinc was not routinely available at clinics or district hospitals at the time of the study, nor was rotavirus vaccination available. Subsequent to our study, rotavirus vaccination became part of the national Extended Programme of Immunization in November 2009, and oral zinc supplementation has been available at clinics and hospitals in the Western Cape since March 2010.

Hypokalaemia was present in just over half of the children tested. Although there was 
no significant difference between those receiving homemade and sachet ORT solutions, homemade SSS does not contain potassium, which is potentially problematic considering the large number of malnourished children in the group. Primary healthcare management may have to be modified to include oral potassium supplementation in accordance with international trends towards use of fluid contaning more potassium for malnourished children with diarrhoea. ${ }^{[24]}$

\section{Study limitations}

There is potential bias in this convenience sample, but it was not possible to systematically collect data on each child owing to the high turnover in the ward and lack of availability of the interviewer on each day. For this reason it was also not possible to use exact rehydrated/ discharge weight when calculating the WAZ. Children who were successfully managed in the community with oral rehydration are not represented, and neither are the sickest children, who would have been transferred directly to the paediatric wards or intensive care unit. This may have affected deductions regarding incorrect mixing of ORT and its impact on electrolyte disturbances.

\section{Conclusions}

It is encouraging that with promotion of SSS, most caregivers in our setting gave ORT. However, many mixed it incorrectly, and they often did not give additional fluid volumes and/or interrupted or replaced milk feeds. There is often a delay in seeking primary care, and most healthcare providers do not give appropriate advice. The majority of children were formula fed and many had suboptimal access to water and sanitation. All these aspects of the WHO CDD programme and IMCI therefore need further promotion in our community and at primary care level, possibly through emphasis on IMCI training and use of messages in the Road-to-Health booklet, and through continuing medical education opportunities for GPs. Many hospitalised children with diarrhoea are malnourished, particularly HIV-infected children. Attention must be paid to nutritional rehabilitation of children presenting with diarrhoea, including continuing feeds during the episode and promotion of additional feeds on discharge. Children with HIV infection are at an increased risk of diarrhoeal disease and malnutrition, and HIV exposure appears to increase the risk of early presentation with diarrhoea. This warrants further study.

Acknowledgements. We thank Abigail Makwenda for data collection and Professor Ben Marais for review of the manuscript.

\section{References}

1. Black RE, Cousens S, Johnson HL. Global, regional, and national causes of child mortality in 2008: A systematic analysis. Lancet 2010;375(9730):1969-1987. [http://dx.doi.org/10.1016/S0140-6736(10)60549-1]

2. Santosham M, Chandran A, Fitzwater C, et al. Progress and barriers for the control of diarrhoeal disease. Lancet 2010;376(9734):63-67. [http://dx.doi. org/10.1016/S0140-6736(10)60356-X]

3. Medical Research Council of South Africa. Burden of Disease Report. http:// www.mrc.ac.za/policybriefs/childmortality.pdf (accessed 22 April 2012)

4. Bhutta ZA, Chopra M, Axelson $\mathrm{H}$, et al. Countdown to 2015 decade report (2000-10): Taking stock of maternal newborn and child survival. Lancet 2010;375(9730):2032-2044. [http://dx.doi.org/10.1016/S0140-6736(10)60676-2]
5. Forsberg BC, Petzold MG, Tomson G et al. Diarrhoea case management in low- and middle-income countries - an unfinished agenda. Bull World Health Organ 2007;85(1):42-48.

6. World Health Organization/UNICEF. Diarrhoea: Why children are still dying and what can be done. Geneva/New York: WHO, 2009. http://www.who.int/ child_adolescent_health/documents/9789241598415/en/index.html (accessed 22 April 2012).

7. Rudan I, Arifeen SE, Black RE, et al. Childhood pneumonia and diarrhoea: Setting our priorities right. Lancet Infect Dis 2007;7(1):56-61. [http://dx.doi. org/10.1016/S1473-3099(06)70687-9]

8. World Health Organization. http://www.who.int/child_adolescent_health/ topics/prevention_care/child/imci/en/index.html (accessed 22 April 2012).

9. Johnson S, Hendson $\mathrm{W}$, Crewe-Brown $\mathrm{H}$, et al. Effect of human immunodeficiency virus infection on episodes of diarrhea among children in South Africa. Pediatr Infect Dis J 2000;19(10):972-999. [http://dx.doi. org/10.1097/00006454-200010000-00007]

10. Chagan MK, Kauchali S. Comorbidities and mortality among children hospitalized with diarrheal disease in an area of high prevalence of human immunodeficiency virus infection. Pediatr Infect Dis J 2006;25(4):333-338. [http://dx.doi.org/10.1097/01.inf.0000207400.93627.4c]

11. Taha TE, Graham SM, Kumwenda NI, et al. Morbidity among human immunodeficiency virus-1-infected and -uninfected African children. Pediatrics 2000;106(6):77-85. [http://dx.doi.org/10.1542/peds.106.6.e77]

12. Mussi-Pinhata MM, Freimanis L, Aparecida Y, et al. Infectious disease morbidity among young HIV-1-exposed but uninfected infants in Latin American and Caribbean countries: The National Institute of Child Health and Human Development International Site Development Initiative Perinatal Study. Pediatrics 2007:119(3):e694-e704. [http://dx.doi.org/10.1542/peds.2006-1856]

13. Marinda E, Humphrey JH, Iliff PJ, et al. Child mortality according to maternal and infant HIV status in Zimbabwe. Pediatr Infect Dis J 2007;26(6):519-526. [http://dx.doi.org/10.1097/01.inf.0000264527.69954.4c]

14. Newell ML, Coovadia H, Cortina-Borja M, et al. Mortality of infected and uninfected infants born to HIV-infected mothers in Africa: A pooled analysis. Lancet 2004;364(9441):1236-1243. [http://dx.doi.org/10.1016/S01406736(04)/7140-7]

15. Wittenberg DF, Loening WE. Diarrhoea is a nutritional disease. S Afr Med J 1989;76(9):476-478

16. Labadarios D, ed. The National Food Consumption Survey - Fortification Baseline (NFCS-FB): The Knowledge, Attitude, Behaviour and Procurement Regarding Fortified Foods, a Measure of Hunger and Anthopometric and Selected Micronutrient Status of Children Aged 1-9 Years and Women of ChildBearing Age: South Africa 2005. Pretoria: Directorate: Nutrition, Department of Bearing Age:

17. Department of Health. The National HIV and Syphilis Antenatal Seroprevalence Survey in South Africa 2007. Pretoria: Directorate: Health Systems Research, Research Coordination and Epidemiology, Department of Health 2008. http://www.doh.gov.za/docs/reports (accessed 22 April 2012).

18. South Africa Demographic and Health Survey. http://www.doh.gov.za/facts/ sadhs2003/part1.pdf (accessed 22 April 2012)

19. Huskisson JM, Feulbach S. A profile of the socio-demographic background of children admitted with acute diarrhea to the Red Cross Children's Hospital, Cape Town. Curationis 1995;18(1):34-36.

20. Dippenaar H, Joubert G, Nel R, et al. Home-made sugar-salt solution for oral rehydration: Knowledge of mothers and caregivers. South African Family Practice 2005;47(2):51-53.

21. Bhutta ZA, Ahmed T, Black RE. What works? Intervention for maternal and child undernutrition and survival. Lancet 2008:371(9610):417-440. [http:// dx.doi.org/10.1016/S0140-6736(07)61693-6]

22. WHO Collaborative Study Team on the Role of Breastfeeding on the Prevention of Infant Mortality. Effect of breastfeeding on infant and child mortality due to infectious diseases in less developed countries: A pooled analysis. Lancet 2000;355(9202):451-455. [http://dx.doi.org/10.1016/S0140-6736(00)82011-5]

23. Fewtrell L, Kaufmann RB, Kay D, et al. Water, sanitation, and hygiene intervention to reduce diarrhea in less developed countries: A systematic review and metaanalysis. Lancet Infect Dis 2005;5(1):42-52. [http://dx.doi. org/10.1016/S1473-3099(04)01253-8]

24. Alam NH, Hamadani JD, Dewan N, et al. Efficacy and safety of modified ora rehydration solution (Resomal) in the treatment of severely malnourished children with watery diarrhea. J Pediatr 2003;143(5):614-618. [http://dx.doi. org/10.1067/S0022-3476(03)00500-6 